\title{
Trapping Hydrogen Atoms From a Neon-Gas Matrix: A Theoretical Simulation
}

\section{Citation}

Bovino, S., Peng Zhang, Vasili A. Kharchenko, and Alexander Dalgarno. 2009. Trapping hydrogen atoms from a neon-gas matrix: a theoretical simulation. Journal of Chemical Physics 131(5): 054302.

\section{Published Version}

doi:10.1063/1.3180822

\section{Permanent link}

http://nrs.harvard.edu/urn-3:HUL.InstRepos:5142875

\section{Terms of Use}

This article was downloaded from Harvard University's DASH repository, and is made available under the terms and conditions applicable to Open Access Policy Articles, as set forth at http:// nrs.harvard.edu/urn-3:HUL.InstRepos:dash.current.terms-of-use\#OAP

\section{Share Your Story}

The Harvard community has made this article openly available.

Please share how this access benefits you. Submit a story.

\section{Accessibility}




\title{
Trapping hydrogen atoms from a neon-gas matrix: A theoretical simulation
}

\author{
S. Bovino, ${ }^{1}$ P. Zhang, ${ }^{1, a)}$ V. Kharchenko, ${ }^{1,2}$ and A. Dalgarno ${ }^{1, b)}$ \\ ${ }^{1}$ Institute for Theoretical Atomic, Molecular and Optical Physics, Harvard-Smithsonian Center for \\ Astrophysics, Harvard University, Cambridge, Massachusetts 02138, USA \\ ${ }^{2}$ Department of Physics, University of Connecticut, Storrs, Connecticut 06269, USA
}

(Received 23 April 2009; accepted 25 June 2009; published online 4 August 2009)

\begin{abstract}
Hydrogen is of critical importance in atomic and molecular physics and the development of a simple and efficient technique for trapping cold and ultracold hydrogen atoms would be a significant advance. In this study we simulate a recently proposed trap-loading mechanism for trapping hydrogen atoms released from a neon matrix. Accurate $a b$ initio quantum calculations are reported of the neon-hydrogen interaction potential and the energy- and angular-dependent elastic scattering cross sections that control the energy transfer of initially cold atoms are obtained. They are then used to construct the Boltzmann kinetic equation, describing the energy relaxation process. Numerical solutions of the Boltzmann equation predict the time evolution of the hydrogen energy distribution function. Based on the simulations we discuss the prospects of the technique. (C) 2009 American Institute of Physics. [DOI: 10.1063/1.3180822]
\end{abstract}

\section{INTRODUCTION}

In the last decade there have been intense efforts to cool atoms leading to the study of states of the matter such as Bose-Einstein condensates and Bardeen-Cooper-Schrieffer behavior in dilute gases. ${ }^{1,2}$ After the advent of buffer-gas loading ${ }^{3}$ many atomic species have been trapped and cooled to ultracold temperatures. Hydrogen is an exception because the current techniques suffer from a variety of experimental limitations and high technical cost. ${ }^{4}$ However, ultracold hydrogen is very important in the advanced spectroscopy field and can offer high precision measurements of the Rydberg constant. ${ }^{5}$ Furthermore, it can lead to the development of highly stable atomic clocks, and it is critical for comparison with cold antihydrogen atoms which are of fundamental interest in tests of $C P T$ symmetry. ${ }^{6,7}$

Recently an interesting proposal ${ }^{8}$ has been advanced for using a trap-loading technique to capture hydrogen atoms released from a solid neon matrix, grown in a cell that contains a cold sapphire substrate. It consists of magnetically capturing the low energy fraction of the released paragmagnetic hydrogen atoms while the host $(\mathrm{Ne})$ atoms stick to the walls. The binding energy of hydrogen to the walls is very low. The main problem is the continuous thermalization of the low energy trapped atoms by the neon, which acts as a temporary buffer gas and causes rapid evaporation of the sample. An approximate estimate of the $\mathrm{H}$ atom energy relaxation has been given in the proposal based on artificial $\mathrm{Ne}-\mathrm{H}$ scattering cross sections and simplified kinetic theory.

The proposed trapping technique depends essentially on the energy transfer rate from the thermal bath to the hydrogen atoms which is the inverse problem of fast atoms relaxing in a thermal bath. ${ }^{9-11}$ Both of these processes are determined by the energy exchange between the projectile

\footnotetext{
${ }^{a)}$ Electronic mail: pezhang@cfa.harvard.edu.

b) Author to whom correspondence should be addressed. Electronic mail: adalgarno@cfa.harvard.edu.
}

particles and the thermostat gas and governed by the same kinetic theory, the classical linear Boltzmann equation. Most of the temperature range in the proposed experiment is in the classical or quasiclassical regime of atomic motion and the classical kinetic Boltzmann equation should be adequate for a description of the energy transfer processes.

\section{THEORY}

\section{A. Elastic cross sections}

The energy relaxation of hydrogen atoms in neon gas occurs due to elastic collisions. The radial part of the Schrödinger equation for center of mass (CM) scattering energy $\varepsilon$ satisfies the Schrödinger equation,

$$
\left\{\frac{d^{2}}{d R^{2}}+k^{2}-U(R)-\frac{\ell(\ell+1)}{R^{2}}\right\} u_{\ell}(R)=0,
$$

where $\mu$ is the reduced mass, $\ell$ is the angular momentum, $R$ is the internuclear distance, $U(R)=2 \mu \mathrm{V}(\mathrm{R}) / \hbar^{2}$ is the molecular potential, and $k^{2}=2 \mu \varepsilon / \hbar^{2}$. The asymptotic solution of Eq. (1) can be written in terms of the spherical Bessel $\left(j_{\ell}\right)$ and Neumann $\left(n_{\ell}\right)$ functions,

$$
u_{\ell}(R)=k R\left[j_{\ell}(k R) \cos \delta_{\ell}-n_{\ell}(k R) \sin \delta_{\ell}\right],
$$

where $\delta_{\ell}$ is the phase shift. At large $R$,

$$
u_{\ell}(R)=\sin \left(k R-\ell \pi / 2+\delta_{\ell}\right),
$$

and the differential cross section is given by

$$
\frac{d \sigma(\varepsilon)}{d \Omega}=\frac{1}{k^{2}}\left|\sum_{\ell=0}^{\infty}(2 \ell+1) \sin \delta_{\ell} e^{\mathrm{i} \delta_{\ell}} P_{\ell}(\cos \chi)\right|^{2},
$$

where $P_{\ell}$ denotes the $\ell$ th Legendre polynomial and $\chi$ is the $\mathrm{CM}$ scattering angle. Atomic units are used throughout this paper. 


\section{B. Kernel of Boltzmann equation}

The energy evolution of atoms due to elastic and inelastic collisions with a uniform bath gas has been considered by Kharchenko et al. ${ }^{10,11}$ The rate of energy transfer of the projectile atoms from initial energy $E^{\prime}$ to final energy $E$ in the laboratory frame (LF) is given by the kernel of the Boltzmann equation $B\left(E \mid E^{\prime}\right)$. In a uniform bath gas, whose density is much higher than that of the projectile atoms, collisions and energy exchange between projectile particles is negligible and the energy distribution $f(E, t)$ of the projectile can be described by the linear Boltzmann equation,

$$
\begin{aligned}
\frac{\partial}{\partial t} f(E, t)= & \int B\left(E \mid E^{\prime}\right) f\left(E^{\prime}, t\right) d E^{\prime}-f(E, t) \int B\left(E^{\prime} \mid E\right) d E^{\prime} \\
& -\zeta(E) f(E, t)+Q(E, t),
\end{aligned}
$$

where $Q(E, t)$ is the rate of production of the atoms with energy $E$ and $\zeta(E, t)$ is the rate of the sink reactions that remove them. For binary elastic and inelastic collisions, $B\left(E \mid E^{\prime}\right)$ can be derived analytically through the double differential cross section $d^{2} \sigma / d \Omega d \varepsilon$ (Refs. 10 and 11), and in the case of elastic scattering, $d^{2} \sigma / d \varepsilon d \Omega=\delta\left(\varepsilon-\varepsilon^{\prime}\right) d \sigma d \Omega$ and the energy relaxation $B\left(E \mid E^{\prime}\right)$ can be calculated using the differential cross section given by Eq. (4),

$$
B\left(E \mid E^{\prime}\right)=\frac{n_{b} m^{3 / 2}}{2 \sqrt{2} \pi \mu^{2}} \sqrt{E} \int \frac{d \sigma}{d \Omega} \rho\left(\mathbf{p}_{b}^{\prime}\right) d \mathbf{p}_{b}^{\prime} d \Omega_{\mathbf{p}} d \Omega_{\mathbf{p}^{\prime}},
$$

where $m$ is the mass of the projectile, $\Omega_{\mathbf{p}^{\prime}}$ and $\Omega_{\mathbf{p}}$ are the solid angles of the initial and final momenta in the LF for fixed $\left|\mathbf{p}^{\prime}\right|=\sqrt{2 m E^{\prime}}$ and $|\mathbf{p}|=\sqrt{2 m E}, n_{b}$ is the density of the bath gas, and $\rho\left(\mathbf{p}_{b}^{\prime}\right)$ is the Maxwellian-Boltzmann distribution function of the bath gas. The solution of Eq. (5) may be written as

$$
f(E, t)=\int_{0}^{t} \int_{0}^{\infty} K\left(E, t \mid E^{\prime}, t^{\prime}\right) Q\left(E^{\prime}, t^{\prime}\right) d E^{\prime} d t^{\prime},
$$

where the propagator $K\left(E, t \mid E^{\prime}, t^{\prime}\right)$ describes the energy evolution of the projectile atoms created at the time $t^{\prime}$ with energy $E^{\prime}$. The propagator can be obtained from the solution of Eq. (5) in which the source function $Q\left(E^{\prime}, t^{\prime}\right)$ is a delta function $\delta\left(E-E^{\prime}\right) \delta\left(t-t^{\prime}\right)$ and there is no sink reaction to remove the projectile. If the initial function $f\left(E^{\prime}, t^{\prime}=0\right)$ is given, $Q\left(E^{\prime}, t^{\prime}\right)=f\left(E^{\prime}, t^{\prime}=0\right) \delta\left(t-t^{\prime}\right), f(E, t)$ at a later time $t$ may be calculated from the propagator $K\left(E, t \mid E^{\prime}, t^{\prime}\right)$ according to

$$
f(E, t)=\int K\left(E, t \mid E^{\prime}, 0\right) f\left(E^{\prime}, 0\right) d E^{\prime} .
$$

Equation (8) can be solved numerically ${ }^{12,13}$ by defining the function $f(E, t)$ in a given energy range $\left[0, E_{\max }\right]$ and using a discretized kinetic equation for the kernel, defined as a $N$ $\times N$ matrix ${ }^{14}$ with the energy grid of $\delta E$,

$$
A_{i j}=\delta E\left[B(i \delta E \mid j \delta E)-\delta_{i j} \omega(i \delta E)\right],
$$

where $\delta_{i j}$ is the Kronecker delta and

$$
\omega(E)=\int_{0}^{\infty} B\left(E \mid E^{\prime}\right) d E^{\prime} .
$$

In the present study, $E_{\max }=0.5 \mathrm{eV}$ and there are 20 energy grid points within $1 k T$. The time-dependent distribution function is generated from the initial distribution function $f(E, 0)$,

$$
f(E, t)=e^{t \mathbf{A}} f(E, 0),
$$

and the time-dependent average translational energy is obtained from

$$
\langle E(t)\rangle=\int f(E, t) E d E .
$$

For an arbitrary time-dependent bath gas density, no general analytical expression for the solution of Boltzmann equation can be obtained. Nevertheless, a solution in closed form may be written for specific time dependence. Thus, for a timedependent density $N(t)=g(t) N(t=0)$, Eq. (11) can be modified to a form with effective time $\tau=\int_{0}^{t} d t^{\prime} g\left(t^{\prime}\right)$,

$$
f(E, t)=e^{\tau \mathbf{A}_{0}} f(E, 0),
$$

where $g(t)$ describes the time variation of the relative bath gas density and $\mathbf{A}_{0}$ is constructed with the density at $t=0$. No sink reaction is involved in the $\mathrm{H}$ trapping process and $\zeta(E, t)=0$.

\section{Molecular potential: Ab initio calculations}

The interaction potential between $\mathrm{Ne}$ and $\mathrm{H}$ atoms has been evaluated using the partially spin restricted coupled cluster single and double ${ }^{15}$ method, augmented with triple excitations noniteratively, ${ }^{16}$ denoted as ROHF-RCCSD(T). All electrons were correlated in the $\operatorname{RCCSD}(\mathrm{T})$ calculation. A series of Dunning's augmented polarized core/valence aug-cc-pCV $n \mathrm{Z}$ basis sets was used in the $a b$ initio calculation $(n=\mathrm{Q}, 5,6)$. Basis set superposition errors were corrected using the standard counterpoise approach of Boys and Bernardi. ${ }^{17}$ The final potential energies were extrapolated to the complete basis set limit by using the mixed exponential/ Gaussian function, ${ }^{18}$

$$
E(n)=E_{\mathrm{CBS}}+B e^{-(n-1)}+C e^{-(n-1)^{2}} .
$$

The MOLPRO2006.1 suite of programs ${ }^{19}$ was used throughout these electronic structure calculations.

The computed potential energies at selected internuclear distances $R$ were then fitted to an analytical expression of the Degli Espositi-Werner ${ }^{20}$ form composed of the short-range term $\left(V_{\mathrm{sh}}\right)$ and the asymptotic long-range part $\left(V_{\mathrm{as}}\right)$,

$$
V(R)=V_{\mathrm{sh}}(R)+V_{\text {as }}(R),
$$

where

$$
V_{\mathrm{sh}}(R)=\sum_{i=0}^{8} a_{i} R^{i} e^{-\alpha(R-\beta)} .
$$

The long-range part was represented by the damped dispersion term, 


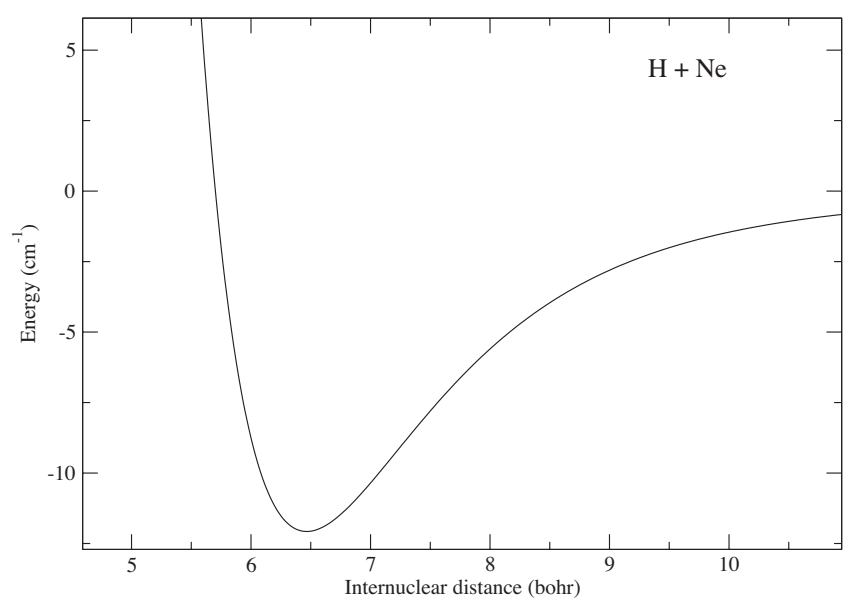

FIG. 1. Diatomic potential of $\mathrm{H}-\mathrm{Ne}$.

$$
V_{\text {as }}(R)=-\frac{1}{2}[1+\tanh (\gamma+\lambda R)] \sum_{n=0}^{4} \frac{C_{2 n+6}}{R^{2 n+6}} .
$$

The nonlinear $\alpha, \beta, \gamma$, and $\lambda$ and the linear $a_{i}$ and $C_{n}$ parameters were optimized using the Levenberg-Marquardt nonlinear least square algorithm with no constraints imposed. The maximum root-mean-square (rms) error is $0.3 \mathrm{~cm}^{-1}$, and the rms error averaged over the internuclear distance does not exceed $0.1 \mathrm{~cm}^{-1}$.

\section{RESULTS AND DISCUSSION}

\section{A. Potential energy curve}

The calculated ground state potential energy curve is shown in Fig. 1 and the corresponding spectroscopic data are listed in Table I with other available experimental ${ }^{21}$ and theoretical $^{22-27}$ results. The system is weakly bound and shows a minimum around 6.47 bohrs. The long-range dispersion coefficient $C_{6}=5.80$ derived from the $a b$ initio potential agrees closely with other theoretical values ranging from 5.688 to 6.2 and obtained by the dipole oscillator strength distribution, ${ }^{23} \mathrm{MBPT}^{22}$ and linear response density functional theory ${ }^{24}$ methods. One bound state with energy of $-0.035 \mathrm{~cm}^{-1}$ relative to the dissociation limit is found for the system with rotational angular momentum $J=0$ corresponding to masses of 19.9924401759 and $1.007825 \mathrm{amu}$ for $\mathrm{Ne}$ and $\mathrm{H}$, respectively.

TABLE I. Spectroscopic data for the diatomic molecule HNe.

\begin{tabular}{lccc}
\hline \hline $\begin{array}{c}R_{e} \\
(\text { bohr })\end{array}$ & $\begin{array}{c}D_{e} \\
(\mathrm{meV})\end{array}$ & $\begin{array}{c}C_{6} \\
(\mathrm{a} . \mathrm{u} .)\end{array}$ & \\
\hline 6.466 & 1.497 & 5.80 & Present \\
6.54 & 1.346 & & 25 \\
6.48 & 1.49 & & 26 \\
6.42 & 1.46 & & 21 (expt.) \\
6.52 & 1.36 & $5.71 \pm 0.07$ & 27 \\
& & 6.227 & 23 \\
& & 5.688 & 22 \\
\hline \hline
\end{tabular}

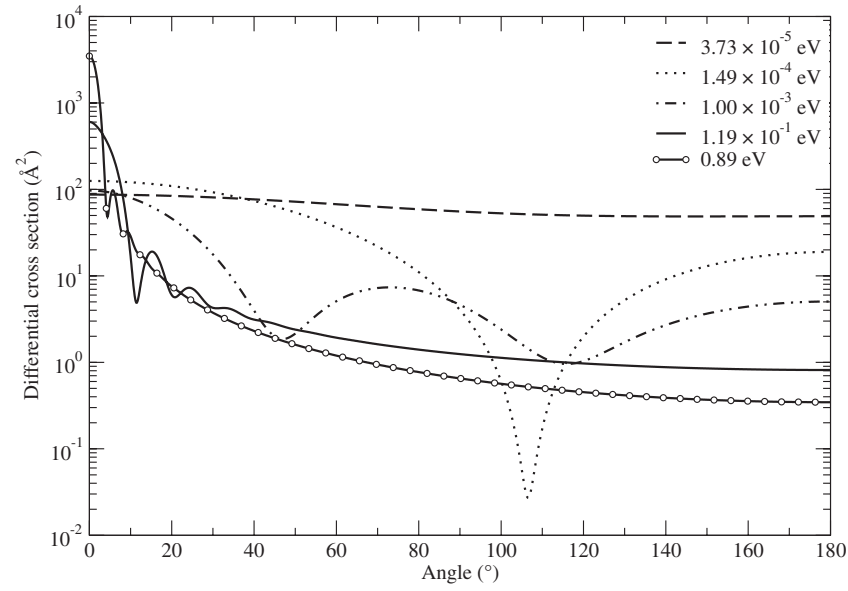

FIG. 2. Differential cross sections at selected CM collision energies $\varepsilon$.

\section{B. Elastic differential and total cross sections}

The calculated differential cross sections at selected energies are shown in Fig. 2. The system starts to enter the cold and ultracold regimes for collision energies below $10^{-3} \mathrm{eV}$. For energies smaller than $10^{-5} \mathrm{eV} s$-wave scattering dominates and the scattering length $a$ may be obtained by fitting the effective range formula, ${ }^{28}$

$$
k \cot \delta_{0}=-\frac{1}{a}+\frac{1}{2} r_{e} k^{2}+O\left(k^{3}\right) .
$$

The derived $a$ and $r_{e}$ are 50.3 and 13.6 a.u., respectively. With increasing scattering energies more partial waves contribute and the differential cross sections demonstrate a detailed angular dependence which determines the energy transfer process. With further increase in energy, small angle scattering dominates.

The total and the momentum transfer cross sections as a function of the projectile energy are shown in Fig. 3. The momentum transfer cross section is defined as

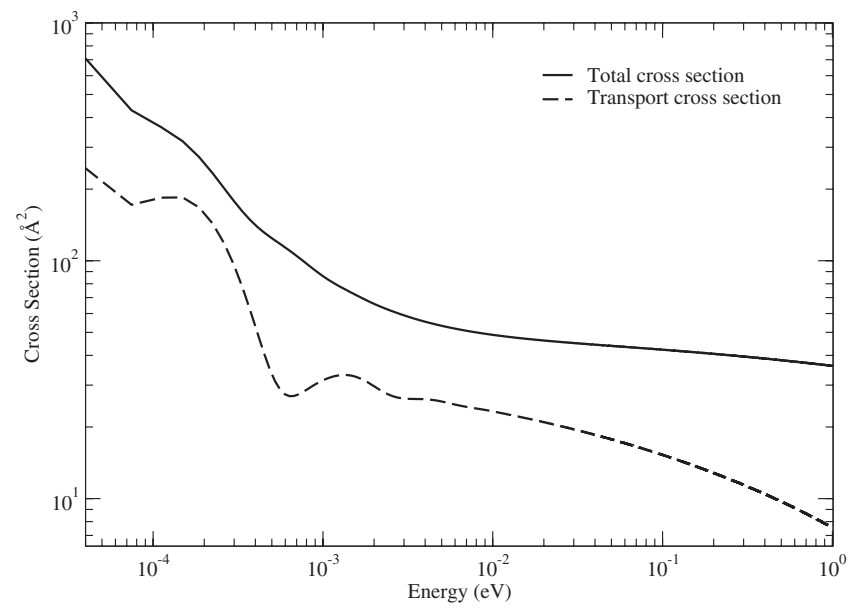

FIG. 3. Total and transport cross sections as a function of CM collision energy. 


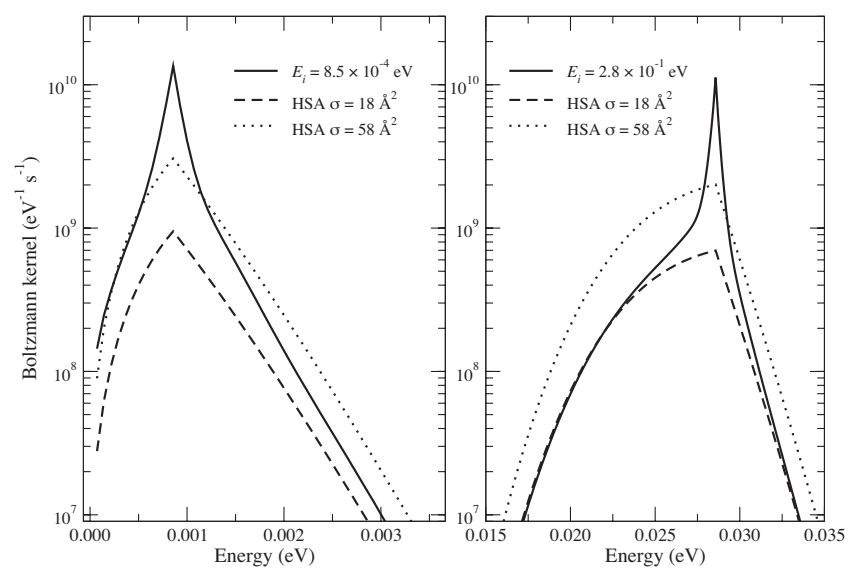

FIG. 4. The exact kernels of the Boltzmann equation at two initial LF energies of $E_{i}=8.5 \times 10^{-5}$ and $E_{i}=2.8 \times 10^{-1}$ as a function of the final LF energy (solid line) and the kernels calculated using HSA with constant cross sections of $18 \AA^{2}$ (dashed line) and $58 \AA^{2}$ (dotted line) in a Ne bath gas with temperature $T=15 \mathrm{~K}$ and a density of $n=1.0 \times 10^{16} \mathrm{~cm}^{-3}$.

$$
\sigma_{\text {transport }}(\varepsilon)=\int \frac{d \sigma(\varepsilon, \chi)}{d \Omega}(1-\cos \chi) d \Omega,
$$

where $d \sigma(\varepsilon, \chi) / d \Omega$ is the differential cross section for scattering into the solid angle $d \Omega$ given by Eq. (4). The classical diffusion coefficient $D_{A B}$ can be evaluated from the momentum transfer cross sections,

$$
D_{A B}=\frac{3 k_{B} T}{16 \mu \Omega_{A, B}} \frac{1}{\left(n_{A}+n_{B}\right)},
$$

where the transport integral $\Omega_{A, B}$ is given in terms of binary collisions between species A and B by

$$
\Omega_{A B}=\frac{2}{\sqrt{\pi \mu}}\left(\frac{1}{2 k_{B} T}\right)^{5 / 2} \int_{0}^{\infty} \varepsilon^{2} \exp \left(-\frac{\varepsilon}{k_{B} T}\right) \sigma(\varepsilon) d \varepsilon,
$$

where $k_{B}$ is the Boltzmann constant, $\sigma(\varepsilon)$ is given by Eq. (19), and $\left(n_{A}+n_{B}\right)$ is the total molecular number density. $D_{\mathrm{HNe}}$ can be reproduced within $1 \%$ by the following expressions:

$$
\begin{aligned}
D_{\mathrm{HNe}}= & \frac{1 \times 10^{16}}{n_{\mathrm{H}}+n_{\mathrm{Ne}}} \times\left[39.505-13.817 \times e^{-0.046561 T}\right. \\
& \left.-33.071 \times e^{-0.55208 T}\right]\left(\mathrm{cm}^{2} \mathrm{~s}^{-1}\right), \quad T \leq 40 \mathrm{~K}, \\
D_{\mathrm{HNe}}= & \frac{1 \times 10^{16}}{n_{\mathrm{H}}+n_{\mathrm{Ne}}} \times[38.641-0.011209 \times T+1.1869 \\
& \times 10^{-7} \times T^{2.5624}+18.681 \times \ln (0.0082299 T) \\
& \left.+29.29 \times e^{-0.011038 T}\right]\left(\mathrm{cm}^{2} \mathrm{~s}^{-1}\right), \quad T>40 \mathrm{~K} .
\end{aligned}
$$

\section{Energy transfer kinetics}

The calculated differential cross sections were used to construct $B\left(E \mid E^{\prime}\right)$ at characteristic bath gas temperatures of 10 and $15 \mathrm{~K}$ with various densities of the Ne gas. In Fig. 4 we show the kernel at $15 \mathrm{~K}$ for a density of $10^{15} \mathrm{~cm}^{-3}$ for initial energies of $8.5 \times 10^{-4}$ and $0.28 \mathrm{eV}$. At both energies,

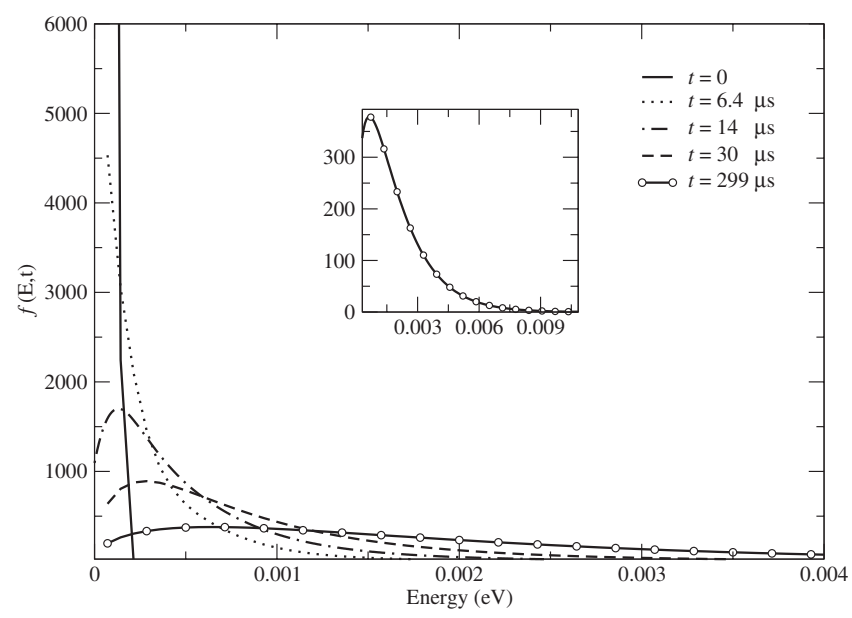

FIG. 5. Translational energy distribution functions of hydrogen atoms at selected relaxation times in a Ne bath gas with temperature $T=15 \mathrm{~K}$ and a density of $10^{15} \mathrm{~cm}^{-3}$. The energy distribution function at $t=299 \mu \mathrm{s}$ is shown in the embedded picture and is overlapped with the theoretical Maxwell-Boltzmann distribution.

sharp maxima occur at $E \sim E^{\prime}$ because collisions are dominated by small angle scattering involving small energy losses.

In applications the hard-sphere approximation (HSA) is often adopted because of its analytical simplicity. Although it lacks angular and energy dependence, an appropriately selected HS cross section provides a realistic account of the physical processes. We found that a HS cross section of $18 \AA^{2}$ reproduces the kernel of the Boltzmann equation at an initial LF energy of $0.28 \mathrm{eV}$, whereas the kinetics of the thermalization were recovered by a HS cross section of $58 \AA^{2}$. The larger HS cross section for the thermalization is a consequence of the absence of angular dependence and the suppression of the peaks in the kernel of the Boltzmann equation obtained with the HSA, indicated in Fig. 4. At the lower energy of $8.5 \times 10^{-4} \mathrm{eV}$, the difference between the HSA kernel and the purely quantum mechanic kernel is less because of the slower variation with scattering angle.

In the proposed experiment the hydrogen is initially held in a magnetic trap with a thermal energy of about $1 \mathrm{~K}$. The hydrogen atoms are heated through collisions with the flowing neon gas at temperatures between 13 and $17 \mathrm{~K}^{8}$ The density of the neon gas is much larger than that of the hydrogen atoms and decays exponentially with time. The feasibility of this trapping proposal depends on the energy transfer rate from neon gas to hydrogen. To gain a better understanding of the kinetics, we investigated the energy transfer process at different bath gas densities and temperatures.

The energy distribution functions of hydrogen atoms were obtained numerically according to Eq. (11). In Fig. 5 we present the translational energy distribution functions at selected times for hydrogen atoms in neon gas with a density of $10^{15} \mathrm{~cm}^{-3}$ and a temperature of $15 \mathrm{~K}$. At $t=0$ the energy distribution function of hydrogen atoms is a delta function peaked at $1 \mathrm{~K}$. As time progresses its amplitude at high energies increases only slowly due to the small cross sections. Gradually the distribution function assumes a Maxwellian- 

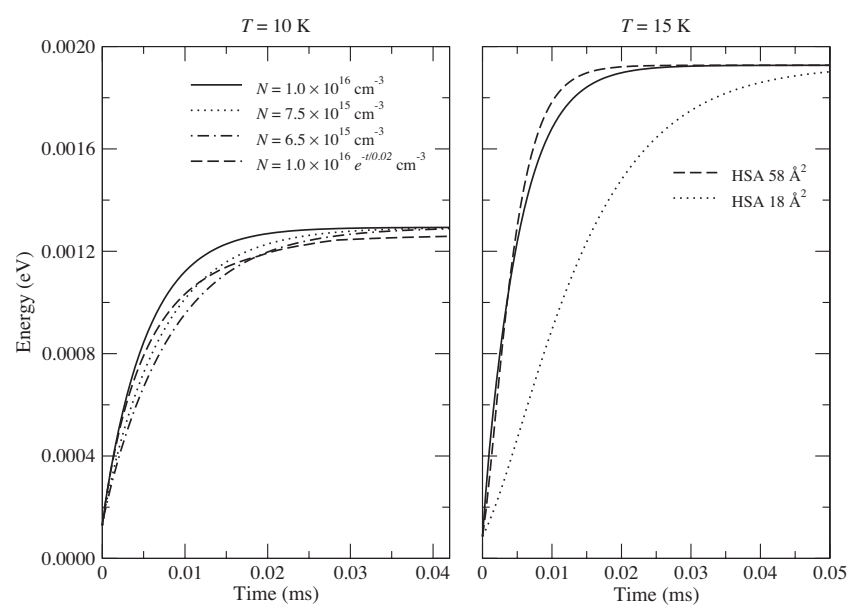

FIG. 6. Average kinetic energy evolution of $\mathrm{H}$ atoms vs the relaxation time in milliseconds in a $\mathrm{Ne}$ bath gas with a density of $10^{16} \mathrm{~cm}^{-3}$ and temperatures of $T=10 \mathrm{~K}$ and $T=15 \mathrm{~K}$ (solid lines). The corresponding average kinetic energy evolution calculated using HSA with two HS cross sections of $18 \AA^{2}$ (dotted line) and $58 \AA^{2}$ (dashed line) in the Ne bath gas with $T$ $=15 \mathrm{~K}$ is given in the right panel. The dashed line in the left panel describes the average kinetic energy relaxation in a bath gas with a time-dependent density.

like shape at some effective temperature $T_{\text {eff }}=(2 / 3)$ $\times\left(E_{\mathrm{eff}}(t) / k_{B}\right)$ and then continuously warms to the bath gas temperature with preservation of the Maxwellian shape, a behavior that is consistent with our previous study of the thermalization of nitrogen atoms in helium and argon gases.

In Fig. 6, we present the average kinetic energy of the evolving hydrogen atoms at bath gas temperatures of 10 and $15 \mathrm{~K}$ and at a bath gas density of $10^{16} \mathrm{~cm}^{-3}$. For the timeindependent bath gas density, the dependence of the average kinetic energy on the density can be obtained by a simple scaling of the time by the inverse of the density. The total times to reach the bath gas temperatures are both $0.032 \mathrm{~ms}$, showing a very weak dependence on $T$. In the right-hand side panel in Fig. 6, which shows the mean energy of the ensemble of hydrogen atoms as a function of time in the $15 \mathrm{~K}$ bath gas, we include the energy relaxation curves obtained by HSA with cross sections of 18 and $58 \AA^{2}$. The HS cross section of $58 \AA^{2}$ was obtained by fitting the resulting energy evolution curve to the $a b$ initio curve. A very different relaxation behavior is obtained with the HS cross section of $18 \AA^{2}$, which yields a best fit to the kernels of the Boltzmann equation. The relaxation kinetics from a timedependent density varying as $N(t)=10^{16} e^{-t / t_{0}} \mathrm{~cm}^{-3}$ with $t_{0}$ $=20 \mu \mathrm{s}$ is given in the left-hand side panel. Because of the fast decay of the bath gas density, the relaxation curve flattens and slows down substantially after $10 \mu \mathrm{s}$. Two additional relaxation curves for specific densities of $7.5 \times 10^{15}$ and $6.5 \times 10^{15} \mathrm{~cm}^{-3}$ are presented in the left panel of Fig. 6 . The curves cross the relaxation curve from the timedependent density at, respectively, 10 and $20 \mu$ s. The overall relaxation curve from the time-dependent density can be constructed by connecting the crossing points of the steadystate curves at different densities. The gas density can be obtained from the projectile energy by measuring the Doppler profile ${ }^{9}$ at a given time during the evolution of the velocity distribution.

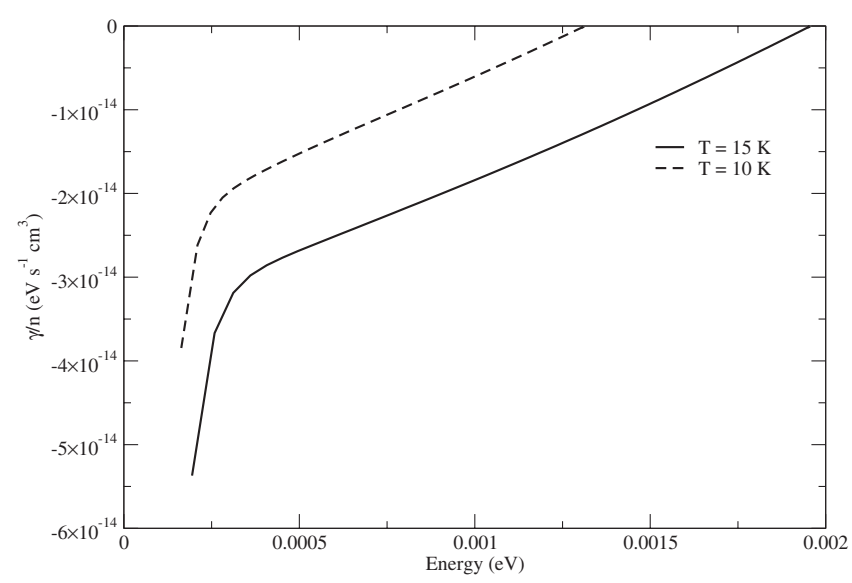

FIG. 7. Density independent energy transfer rate as a function of kinetic energy in eV for two selected temperatures of $10 \mathrm{~K}$ (dashed line) and $15 \mathrm{~K}$ (solid line) for initially cold $\mathrm{H}$ atoms thermalized in a $\mathrm{Ne}$ bath gas.

The energy transfer rate $\gamma=-(d E / d t)\left(1 / n_{b}\right)$ for hydrogen atoms in a neon-gas bath is independent of density. It is shown as a function of translational energy in Fig. 7. The higher the temperature, the faster the energy transfer rate. Between 10 and $15 \mathrm{~K}$ the increase in the energy transfer rate is about $10 \%$.

The idea in the proposed $\mathrm{H}$ trapping experiment is to trap the fraction of the weak field seeker released from an initially prepared Ne matrix whose energy lies below the trap depth. ${ }^{8,29}$ The depth $E_{\text {trap }}$ for a $1 \mathrm{~T}$ trap is $0.67 \mathrm{~K}$ and for a $3 \mathrm{~T}$ trap is $2.01 \mathrm{~K}$. The initial density ratio of $\mathrm{H}$ to $\mathrm{Ne}$ is $\sim 1: 10^{3}$ with a Ne density of $1.22 \times 10^{15} \mathrm{~cm}^{-3}$. Collision energy transfer will remove some of the $\mathrm{H}$ atoms from the trap. In our simulation, the initial $\mathrm{H}$ distributions were taken as part of a $15 \mathrm{~K}$ Maxwell-Boltzmann distribution with a cutoff corresponding to the trap depth. The Ne density decays exponentially with time and can be approximated as ${ }^{29}$

$$
N(t)=N_{0} e^{-t / t_{0}},
$$

where $t_{0}$ is the inverse of the rate of change of the bath gas density, estimated to be less than $60 \mu \mathrm{s}$. Three trial values of 60,40 , and $20 \mu$ s are adopted for our simulation for which we used three initial bath gas densities of $1.22 \times 10^{15}, 0.81$ $\times 10^{15}$, and $0.41 \times 10^{15} \mathrm{~cm}^{-3}$. The calculated trapping efficiencies expressed as percentages of the spin-up hydrogen atoms at times of 60 and $100 \mu$ s are listed in Table II. Multiplication of the percentages by the product of the initial $\mathrm{Ne}$ density, the $\mathrm{H}$ to $\mathrm{Ne}$ density ratio, and the fraction of the initially trapped $\mathrm{H}$ atoms to total number of atoms yields the number density of trapped $\mathrm{H}$ atoms. The factors of 0.28 and 0.74 correspond to the fractions of initially trapped $\mathrm{H}$ atoms in $1 \mathrm{~T}$ and $3 \mathrm{~T}$ traps, respectively. They are given by $\int_{0}^{E_{\text {trap }}} f(E) d E$, where $f(E)$ is the initial Maxwell distribution and normalized to unity. After $100 \mu \mathrm{s}$, the Ne density for all three conditions is less than $10^{14} \mathrm{~cm}^{-3}$, and it continues to decay with a slower change until $1 \mathrm{~ms}$ when the density becomes $10^{11} \mathrm{~cm}^{-3}$. The small Ne density in this time period causes a slow energy transfer between $\mathrm{H}$ and $\mathrm{Ne}$ atoms and a slow decrease in the trapping percentage as shown by the small changes in the trapping percentage from $t=60 \mu$ s to $t=100 \mu \mathrm{s}$. For the fastest decay rate, $t_{0}=20 \mu \mathrm{s}$, the de- 
TABLE II. The trapped percentage of the $\mathrm{H}$ atoms at two relaxation times of $t=60 \mu \mathrm{s}$ and $t=100 \mu \mathrm{s}$ for differential initial Ne bath gas densities $N_{0}$ and decay rates $t_{0}^{-1}$ for two traps with depths of $3 \mathrm{~T}$ and $1 \mathrm{~T}$, respectively.

\begin{tabular}{|c|c|c|c|c|c|c|c|}
\hline & \multirow{2}{*}{$\begin{array}{c}N_{0} \\
\left(\mathrm{~cm}^{-3}\right)\end{array}$} & \multicolumn{3}{|c|}{$t=60 \mu \mathrm{s}$} & \multicolumn{3}{|c|}{$t=100 \mu \mathrm{s}$} \\
\hline & & $t_{0}=60$ & $t_{0}=40$ & $t_{0}=20$ & $t_{0}=60$ & $t_{0}=40$ & $t_{0}=20$ \\
\hline \multirow[t]{3}{*}{$3 \mathrm{~T}$} & $1.22 \times 10^{15}$ & 5.8 & 6.9 & 10.3 & 4.9 & 6.0 & 10.0 \\
\hline & $0.81 \times 10^{15}$ & 8.2 & 9.7 & 14.3 & 6.6 & 8.5 & 14.0 \\
\hline & $0.41 \times 10^{15}$ & 14.4 & 16.7 & 22.7 & 11.7 & 14.6 & 22.1 \\
\hline \multirow[t]{3}{*}{$1 \mathrm{~T}$} & $1.22 \times 10^{15}$ & 1.2 & 1.4 & 2.3 & 1.0 & 1.2 & 2.2 \\
\hline & $0.81 \times 10^{15}$ & 1.7 & 2.1 & 3.3 & 1.4 & 1.8 & 3.2 \\
\hline & $0.41 \times 10^{15}$ & 3.3 & 3.9 & 5.4 & 2.7 & 3.3 & 5.3 \\
\hline
\end{tabular}

crease is $\sim 2 \%$. For the slower density decay rate, the largest decrease does not exceed $20 \%$, and, in general, it is $\sim 15 \%$. Therefore, the further decrease in trapped $\mathrm{H}$ atoms will be less than $10 \%$ even for the slowest density decay rate in our simulations.

The dependence of the trapping percentage on the initial density $N_{0}$ and on the decay rate $t_{0}^{-1}$ is not linear. The trapped percentage increases with the decrease in $N_{0}$, but the number density of trapped $\mathrm{H}$ atoms decreases from $1.22 \times 10^{10}$ to $1.11 \times 10^{10} \mathrm{~cm}^{-3}$ for a 1T trap with $t_{0}=60 \mu \mathrm{s}$. On the other hand, the fastest density decay, $t_{0}=20 \mu \mathrm{s}$, increases the number density of trapped $\mathrm{H}$ atoms by a factor of $\sim 2$ for the same $1 \mathrm{~T}$ trap. For the intermediate decay rate, $t_{0}=40 \mu \mathrm{s}$, the increase is less than $30 \%$ for both of the $3 \mathrm{~T}$ and 1T traps compared to $t_{0}=20 \mu \mathrm{s}$. The deeper trap will also help to retain the $\mathrm{H}$ atoms. Compared to the 1T trap, the 3T trap generally increases the number density of trapped $\mathrm{H}$ atoms by a factor of $\sim 4$ for the same conditions.

It seems there is no optimal condition such that the trapped number density is a maximum with respect to the initial density and decay rate, but the fast density decay and deeper trap will certainly help to trap the $\mathrm{H}$ atoms. Our simulations suggest that a trapped number density on the order of $\sim 10^{9}-10^{8} \mathrm{~cm}^{-3}$ could be achieved. No magnetic field is considered in the Boltzmann equation, but the estimated order of magnitude will not change much.

\section{SUMMARY AND CONCLUSION}

$A b$ initio calculations of the time-dependent thermalization of initially cold hydrogen atoms in a buffer gas of neon atoms were performed. The atomic interaction potential was constructed at the coupled cluster level of theory with extrapolation to the complete basis limit. Elastic differential cross sections were evaluated quantum mechanically. The derived scattering length and effective range are 50.3 and 13.6 a.u., respectively. The diffusion coefficient was evaluated at temperatures between 1 and $1000 \mathrm{~K}$. The kernels of the Boltzmann equation, describing the rate of energy change collisions in the LF, were constructed using the elastic differential cross sections, and the corresponding timedependent solutions of the Boltzmann equation were used to simulate a proposed experiment for trapping hydrogen atoms. A number density of trapped $\mathrm{H}$ atoms on the order of $10^{9} \sim 10^{8} \mathrm{~cm}^{-3}$ was obtained in our simulation. It supports the proposed experiment in which a hydrogen density of the order of $10^{8} \mathrm{~cm}^{-3}$ was estimated, based on approximations and a simplified kinetic theory.

\section{ACKNOWLEDGMENTS}

The research of A.D. and P.Z. was supported by the Chemical Science, Geoscience and Bioscience Division of the Office of Basic Energy Science, Office of Science, U.S. Department of Energy and of V.K. by NASA Grant No. NNX07AR03G. S.B. thanks ITAMP and the HarvardSmithsonian Center for Astrophysics for his participation in their predoctoral program. We are grateful to Professor Claudio Cesar and Dr. Paolo Crivelli for sharing the details of the proposed experiment. The computational resources were provided by the National Center for Atmospheric Research (NCAR).

${ }^{1}$ C. A. Regal, M. Greiner, and D. S. Jin, Phys. Rev. Lett. 92, 040403 (2004).

${ }^{2}$ M. W. Zwierlein, C. A. Stan, C. H. Schunck, S. M. F. Rampach, A. J. Kerman, and W. Ketterle, Phys. Rev. Lett. 92, 120403 (2004).

${ }^{3}$ M. Doyle, B. Friedrich, J. Kim, and D. Patterson, Phys. Rev. A 52, R2515 (1995)

${ }^{4}$ R. de Carvalho, N. Brahms, B. Newman, J. M. Doyle, D. Kleppner, and T. Greytak, Can. J. Phys. 83, 293 (2005).

${ }^{5}$ T. Hansch, Rev. Mod. Phys. 78, 1297 (2006).

${ }^{6}$ G. Andresen, W. Bertsche, A. Boston, P. D. Bowe, C. L. Cesar, S. Chapman, M. Charlton, M. Chartier, A. Deutsch, J. Fajans, M. C. Fujiwara, R. Funakoshi, D. R. Gill, K. Gomberoff, J. S. Hangst, R. S. Hayano, R. Hydomako, M. J. Jenkins, L. V. Jrgensen, L. Kurchaninov, N. Madsen, P. Nolan, K. Olchanski, A. Olin, A. Povilus, F. Robicheaux, E. Sarid, D. M. Silveira, J. W. Storey, H. H. Telle, R. I. Thompson, D. P. van der Werf, J. S. Wurtele, and Y. Yamazaki, Phys. Rev. Lett. 98, 023402 (2007).

${ }^{7}$ G. Gabrielse, P. Larochelle, D. Le Sage, B. Levitt, W. S. Kolthammer, R. McConnell, P. Richerme, J. Wrubel, A. Speck, M. C. George, D. Grzonka, W. Oelert, T. Sefzick, Z. Zhang, A. Carew, D. Comeau, E. A. Hessels, C. H. Storry, M. Weel, and J. Walz, Phys. Rev. Lett. 100, 113001 (2008).

${ }^{8}$ R. Lambo, C. C. Rodegheri, D. M. Silveira, and C. L. Cesar, Phys. Rev. A 76, 061401(R) (2007).

${ }^{9}$ P. Zhang, V. Kharchenko, A. Dalgarno, Y. Matsumi, T. Nakayama, and K. Takahashi, Phys. Rev. Lett. 100, 103001 (2008).

${ }^{10}$ V. Kharchenko, J. T. Tharamel, and A. Dalgarno, J. Atmos. Sol.-Terr. Phys. 59, 107 (1997).

${ }^{11}$ V. Kharchenko, N. Balakrishnan, and A. Dalgarno, J. Atmos. Sol.-Terr. Phys. 60, 95 (1998).

${ }^{12}$ P. Zhang, V. Kharchenko, and A. Dalgarno, Mol. Phys. 105, 1485 (2007).

${ }^{13}$ V. Kharchenko and A. Dalgarno, J. Geophys. Res. 109, D18311 (2004).

${ }^{14}$ I. Oppenheim, K. Schuler, and G. Weiss, Stochastic Processes in Chemical Physics: The Master Equation (MIT Press, Cambridge, MA, 1977). 
${ }^{15}$ P. J. Knowles, C. Hampel, and H.-J. Werner, J. Chem. Phys. 99, 5219 (1993).

${ }^{16}$ J. D. Watts, J. Gauss, and R. J. Bartlett, J. Chem. Phys. 98, 8718 (1993).

${ }^{17}$ S. F. Boys and F. Bernardi, Mol. Phys. 19, 553 (1970).

${ }^{18}$ K. A. Peterson, D. E. Woon, and T. H. Dunning, Jr., J. Chem. Phys. 100, 7410 (1994).

${ }^{19}$ H.-J. Werner, P. J. Knowles, R. Lindh et al., MOLPRO is a package of $a b$ initio programs.

${ }^{20}$ A. Degli Espositi and H.-J. Werner, J. Chem. Phys. 93, 3351 (1990),

${ }^{21}$ N. Hishinuma, J. Chem. Phys. 75, 4960 (1981).

${ }^{22}$ A. J. Thakkar, H. Hettema, and P. E. S. Wormer, J. Chem. Phys. 97, 3252 (1992).
${ }^{23}$ A. Kumar and W. J. Meath, Mol. Phys. 54, 823 (1985).

${ }^{24}$ M. Lein, J. F. Dobson, and E. K. U. Gross, J. Comput. Chem. 20, 12 (1999).

${ }^{25}$ H. Partridge, D. W. Shwenke, and C. W. Bauschlicher, Jr., J. Chem. Phys. 99, 9776 (1993).

${ }^{26}$ K. T. Tang and J. P. Toennies, Chem. Phys. 156, 413 (1991).

${ }^{27}$ T. Kiljunen, J. Eloranta, and H. Kunttu, J. Chem. Phys. 110, 11814 (1999).

${ }^{28}$ R. Côté and A. Dalgarno, Phys. Rev. A 50, 4827 (1994).

${ }^{29}$ P. Crivelli, C. L. Cesar, and R. Lambo, "A new trap loading mechanism for Hydrogen," special issue of Can. J. Phys. (in press). 